TITLE:

\title{
Characterization of Adsorbed Molecular Water on the Surface of a Stretched Polytetrafluoroethylene Tape Analyzed bylH NMR
}

\section{$\operatorname{AUTHOR(S):~}$}

Wakai, Chihiro; Shimoaka, Takafumi; Hasegawa, Takeshi

\section{CITATION:}

Wakai, Chihiro ... [et al]. Characterization of Adsorbed Molecular Water on the Surface of a Stretched Polytetrafluoroethylene Tape Analyzed by1H NMR. Journal of Physical Chemistry B 2016, 120(9): 2538-2543

ISSUE DATE:

2016-02-05

URL:

http://hdl.handle.net/2433/218720

\section{RIGHT:}

This document is the Accepted Manuscript version of a Published Work that appeared in final form in 'Journal of Physical Chemistry B', copyright ( $)$ American Chemical Society after peer review and technical editing by the publisher To access the final edited and published work see http://doi.org/10.1021/acs.jpcb.5b11276; この論文は出版社版であり ません。引用の際には出版社版をご確認ご利用ください。; This is not the published version. Please cite only the published version. 


\title{
Characterization of Adsorbed Molecular Water on the Surface of a Stretched Polytetrafluoroethylene
}

\author{
Tape Analyzed by ${ }^{1} \mathrm{H}$ NMR
}

\author{
Chihiro Wakai, Takafumi Shimoaka, and Takeshi Hasegawa*
}

Institute for Chemical Research, Kyoto University, Uji, Kyoto 611-0011, Japan 
ABSTRACT: A single molecule often exhibits a largely different material character from a bulk matter. Although a perfluoroalkyl (Rf) compound is a representative one, many interests have mostly been devoted to the bulk character only thus far, leaving the single molecular character unclear. Recently, a new theoretical framework, stratified dipole-arrays (SDA) theory, has appeared for comprehensive understanding of $\mathrm{Rf}$ compounds in terms of both single and bulk systems. On this theory, a mechanically stretched polytetrafluoroethylene (PTFE) is expected to exhibit a single-molecular character having dipole-driven properties, which should attract molecular water. In the present study, a stretched PTFE tape is revealed to attract molecular water (not water droplet) in fact, and the adsorbed water molecules are highly restricted in motion by the dipole-dipole interaction studied by using ${ }^{1} \mathrm{H}$ NMR, which agrees with the prediction by the SDA theory.

KEYWORDS: PTFE, a single molecule, SDA theory, molecular water adsorption, ${ }^{1} \mathrm{H}$ NMR, molecular motion 
INTRODUCTION: Comprehensive understanding of the bulk properties of a perfluoroalkyl (Rf) compound represented by the hydrophobicity, a high melting temperature and a low electric permittivity $^{1}$ has long been an unresolved chemical matter, especially when it is tried to be related to the primary chemical structure. Recently, a novel theoretical framework, the stratified dipole-arrays (SDA) theory, ${ }^{2}$ has been proposed to account for the bulk properties in a unified manner on the primary structure. The SDA theory is based on the representative two facts that 1) the $\mathrm{C}-\mathrm{F}$ bond has a large electric dipole moment, ${ }^{3}$ and the molecular interaction is driven by the dipole-dipole interaction (i.e., the orientation effect), ${ }^{4}$ and 2 ) the helical structure about the Rf group induces a spontaneous two-dimensional molecular aggregates, which generates the $\mathrm{Rf}$ specific properties to a "bulk matter." In other words, the strict discrimination of a single Rf group from an aggregate is the key for the comprehensive understanding of $\mathrm{Rf}$ compounds.

Polytetrafluoroethylene (PTFE; known as Teflon ${ }^{\circledR}$ ) is a polymer of a long Rf chain, and the bulky matter comprises fibril aggregates ${ }^{5}$ of the polymer chains. As predicted by the SDA theory, Rf chains with a long chain length are aggregated tightly exhibiting a high melting point of $327{ }^{\circ} \mathrm{C} .{ }^{1,2}$ Since the dipole-dipole interaction is one of the van der Waals forces, ${ }^{4}$ however, the molecular aggregation is much weaker than the covalent and ionic bonds, and the aggregated molecules can be taken apart by applying an external force. For example, when a PTFE tape is mechanically stretched in length, some fibril aggregates are expected to come loosen to yield a single $\mathrm{Rf}$ molecule faced to the air. Since a single $\mathrm{Rf}$ group has many apparent dipole moments along the C-F bonds, the stretched PTFE tape should attract "molecular" water via the dipoledipole interaction.

Here, we have to note that "bulky" water like a water droplet is too large to recognize each dipole moment of a $\mathrm{C}-\mathrm{F}$ bond, $\mu$, since the "summation" of the dipole moments with various 
directions along the $\mathrm{Rf}$ chain, $P=\sum \mu$, is recognized in the "bulk scale," which results in $P \approx 0 .^{3}$ As a result of the disappearance of the polarization density, $P$, the system is changed from the dipole-dipole interaction in the molecular scale to the dispersion-force driven system in the bulky scale. ${ }^{3}$ In this manner, the character of "molecular water" should also be discriminated from the "bulky water" as well as the discrimination of the molecular aggregations of Rf groups. Thus far, the surface property of a bulky matter has extensively been studied on Rf compounds ${ }^{5-}$ ${ }^{10}$; whereas very few properties of a single Rf group have been reported.

Adsorption of molecular water on the surface of a bulky PTFE sheet was reported by Chessick et al. ${ }^{6}$ by measuring an adsorption isotherm in comparison to that on carbon black. They found that the PTFE surface has a hydrophilic site of about $0.75 \%$ of the surface area, which is larger than that of carbon black of $0.15 \%$. The chemical reason of the hydrophilic site on carbon black is attributed to the surface oxide, and the same mechanism is taken into account for PTFE, which seems ambiguous. Fowkes et al. compared gas adsorption of water to argon on a PTFE surface. ${ }^{7}$ They report that the number of water adsorption sites is much less than that of argon, but adsorption of gaseous water on PTFE is more than ten times stronger than argon, which suggests an important point that the adsorption sites should have apparent dipole moment.

To study the molecular interaction between a single $\mathrm{Rf}$ group and molecular water, in the present study, a stretched PTFE tape is employed as an analytical stage, on which the spontaneously adsorbed molecular water is analyzed by using ${ }^{1} \mathrm{H}$ NMR. As a result, a stretched PTFE tape proves to have an apparent molecular adsorption of water on the surface; whereas an un-stretched tape has no adsorbed water as predicted by the SDA theory for both cases. The chemical shift of the NMR peak of the adsorbed-water suggests that the molecular adsorption reflects a dipole environment in the surface of the stretched PTFE tape. Via the analysis of 
molecular motion of the adsorbed water ( $T_{1}$ analysis), the adsorption on the surface is found to be quite strong, which cannot be explained by the dispersion force. In addition, the thermal desorption of water is also analyzed, and the extraordinarily strong adsorption on the stretched tape is confirmed.

In this manner, the molecular interaction between a single $\mathrm{Rf}$ group and a water molecule is found to be totally different from the conventional concept of hydrophobicity or hydrophilicity in the conventional surface chemistry, which always requires bulky water like a water droplet.

\section{MATERIALS AND METHODS}

Materials: A PTFE tape was purchased from AS ONE Corporation (Tokyo, Japan). The tape exhibited only Rf-related bands in an FT-IR spectrum, which guaranteed the purity of the product. For the experiments on a stretched tape, a PTFE tape with $6 \mathrm{~cm}$ in length was 1.5 times stretched in the length direction. Three pieces of the PTFE tape were loosely rolled and put in a NMR tube with 5 mm-o.d. in a stacked manner (Figure 1) to keep the cylindrical shape, so that the shape factor can be zero.

Pure water was obtained by a Yamato (Tokyo, Japan) Autopure WT100U water purifier after passing through a Millipore (Molsheim, France) Elix UV-3 pure-water generator. The electric resistivity of the water was higher than $18.2 \mathrm{M} \Omega \mathrm{cm}$, and the surface tension was 72.5 $\mathrm{mN} \mathrm{m}^{-1}$ at $25{ }^{\circ} \mathrm{C}$, which was measured by using a Kyowa Interface Science Co., Ltd. (Saitama, Japan) DropMaster, DM-501Hy, contact-angle meter. The water-vapor adsorption on the tape was performed at $30^{\circ} \mathrm{C}$ for more than $72 \mathrm{~h}$.

NMR Measurements: The one-dimensional ${ }^{1} \mathrm{H}$ NMR spectra and the spin-lattice relaxation time, $T_{1}$, were measured by using a JEOL (Tokyo, Japan) ECA600 NMR spectrometer (600 $\mathrm{MHz}$. Pure heavy water was used as the external reference to calibrate the chemical shift, and 
the standard signal was set to $4.72 \mathrm{ppm}$ at $30{ }^{\circ} \mathrm{C}$. The FID signal was accumulated 512 times for the spectral collection. The digital resolution was $0.344 \mathrm{~Hz}$. To measure $T_{1}$, the inversion-recovery method $^{11}$ was used. The pulse sequence was $\pi-t_{\operatorname{mix}}-\pi / 2$, where $\pi$ and $\pi / 2$ are the nonselective $180^{\circ}$ and $90^{\circ}$ pulses, respectively, and $t_{\text {mix }}$ is the mixing time. The FID signals were accumulated 256 times for individual eighteen different mixing times, which took 14 hours.

\section{RESULTS AND DISCUSSION}

Adsorption of Water on a stretched PTFE Tape: Figure 2 presents ${ }^{1} \mathrm{H}$ NMR spectra of PTFE tapes with various conditions. The dark-blue curve is a spectrum of an un-stretched tape stored in an ambient air (RH 35\%), which is nearly identical to the spectrum of "dry air only" (without tape; black curve). The strong sharp peak at $3.4 \mathrm{ppm}$ is due to the minute water gas (ca. $1.4 \mathrm{mM}$ ) involved in the temperature-controlling air outside the NMR tube. In general measurements, this peak is invisible because of the weak intensity. In the present study, however, this peak becomes significant after the huge magnification of the ordinate scale. Therefore, the un-stretched PTFE tape accompanies nothing on the surface, which agrees with a common sense that the PTFE surface is water- and oil-repelling. ${ }^{1}$

After the PTFE tape was 1.5 times stretched in length and it was left in the ambient air for about 30 min, the tape was put in the NMR tube, which yielded an NMR spectrum presented by the green curve. This spectrum exhibits an apparent broad peak at ca. $2.0 \mathrm{ppm}$, which is not available in the former two spectra at all. Since the NMR measurements are for ${ }^{1} \mathrm{H}$, PTFE yields no peak in principle irrespective of stretching of the tape. Only a possible reason to generate the new peak is that gaseous molecules in the ambient air adsorb on the surface of the stretched PTFE tape. Since water is the unique chemical species containing proton in the ambient air, this 
newly appeared peak should be assigned to the gaseous water molecules interacted with the PTFE tape.

The reader may be, however, concerned about an impurity that yields the "water peak," which is involved in the PTFE tape. Therefore, just in case, a PTFE tape was stretched in a dry box having no water gas, and the stretched tape was put in an NMR tube in the dry box. The ${ }^{1} \mathrm{H}$ NMR spectrum is presented in Figure S1. The spectrum is nearly identical to the spectrum of dry air (without tape), which straightforwardly implies that the "water peak" is not due to the impurity involved in the tape, but should be attributed to a gaseous water in ambient air.

This spontaneous molecular adsorption may look strange to a common sense that PTFE is hydrophobic. According to the SDA theory, the mechanical stretch makes the molecular fibers disaggregated, which makes the "molecular surface" faced to the air. The surface of a single Rf group has a lot of dipole moments along the $\mathrm{C}-\mathrm{F}$ bonds, and the surface should attract molecular water, since a water molecule also has a large dipole moment. In fact, the large peak-width implies that the molecular motion of water is restricted by a strong interaction.

To confirm the water adsorption, an NMR spectrum of a stretched PTFE tape was measured in a water vapor in the NMR tube after storing in a humid environment for $72 \mathrm{~h}$. The spectrum (orange curve) readily reproduces the peak at the same position, which confirms that the peak is derived from the adsorbed water molecules on the tape surface. To discriminate whether the water molecules are on the PTFE surface or gas in the tube, the tube was vacuumed for $14 \mathrm{~h}$. As a result, the red spectrum appears with a little weaker intensity at $1.5 \mathrm{ppm}$. Of note is that the apparently remained water peak implies that the adsorbed water remains even after the vacuuming. By reducing the pressure, not only the gaseous water, but excess water on the adsorbed water is also removed. Therefore, the new peak position at $1.5 \mathrm{ppm}$ can be recognized 
to be a real position of the directly adsorbed molecular water on PTFE. In order to estimate the amount of the adsorbed water, we have measured a ${ }^{1} \mathrm{H}$ NMR spectrum for a saturated water gas at $30{ }^{\circ} \mathrm{C}$ as an external reference. As a result, the relative amount of water to PTFE was calculated to be $2.6 \times 10^{-2} \mathrm{wt} \%$.

Besides, when a water droplet (bulky water) was put on the same PTFE tapes, the PTFE surface exhibited an apparent hydrophobicity. The contact angle of the water droplet $(4 \mu \mathrm{L})$ was $123 \pm 3^{\circ}$ and $124 \pm 6^{\circ}$ on the un-stretched and stretched PTFE tapes, respectively. This indicates that the bulky water is impervious to the molecular dipole character of PTFE, as predicted by the SDA theory $(P \approx 0)$. Although the contact angle of a water droplet is sometimes influenced by a large extension of the tape because of the surface morphological change, ${ }^{9}$ the surface character can roughly be categorized into hydrophobicity. Contrary to the hydrophobic property of the bulky water, the strong adsorption of the "molecular water" on the stretched tape is particularly of note.

Chemical environment of the adsorbed water: To quantitatively discuss the chemical shift of the adsorbed molecular water, the peak position should accurately be obtained by considering the magnetic susceptibility correction. In the present study, the external reference method is employed. As described in the literature, ${ }^{12}$ the magnetic field in the PTFE tape, $H_{\mathrm{S}}$, is correlated with the volume magnetic susceptibility, $\chi_{\mathrm{s}}$, as follows:

$$
H_{\mathrm{S}}=H_{0}\left[1+\left(\frac{4}{3} \pi-\alpha\right) \chi_{\mathrm{S}}\right]
$$

where $H_{0}$ is the magnetic field in vacuum. In this study, the shape factor, $\alpha$, can be $\alpha=0$, since the three rolled tapes are piled up, so that the sample has a cylindrical shape (Figure 1). In 
the present study, heavy water is employed as the external reference substance. The magnetic field in the reference, $H_{\mathrm{R}}$, is defined in the same manner,

$$
H_{\mathrm{R}}=H_{0}\left(1+\frac{4}{3} \pi \chi_{\mathrm{R}}\right)
$$

using the volume magnetic susceptibility of heavy water, $\chi_{\mathrm{R}}$.

The observed chemical shift, $\delta_{\text {obs }}$, is expressed as follows by considering the Larmor equation $\left(\omega=\gamma \mu_{0} H\right)$ and the two good approximations: $\chi_{\mathrm{R}} \ll 1$ and $\sigma_{\mathrm{R}} \ll 1$.

$$
\begin{aligned}
\delta_{\text {obs }} \equiv & \frac{\omega_{\mathrm{S}}-\omega_{\mathrm{R}}}{\omega_{\mathrm{R}}} \times 10^{6} \\
& =\frac{H_{\mathrm{S}}\left(1-\sigma_{\mathrm{S}}\right)-H_{\mathrm{R}}\left(1-\sigma_{\mathrm{R}}\right)}{H_{\mathrm{R}}\left(1-\sigma_{\mathrm{R}}\right)} \times 10^{6} \\
& =\frac{\left(1+\frac{4}{3} \pi \chi_{\mathrm{S}}\right)\left(1-\sigma_{\mathrm{S}}\right)-\left(1+\frac{4}{3} \pi \chi_{\mathrm{R}}\right)\left(1-\sigma_{\mathrm{R}}\right)}{\left(1+\frac{4}{3} \pi \chi_{\mathrm{R}}\right)\left(1-\sigma_{\mathrm{R}}\right)} \times 10^{6} \\
& \approx \frac{\left(\sigma_{\mathrm{R}}-\sigma_{\mathrm{S}}\right)-\frac{4}{3} \pi\left(\chi_{\mathrm{R}}-\chi_{\mathrm{S}}\right)}{1-\sigma_{\mathrm{R}}+\frac{4}{3} \pi \chi_{\mathrm{R}}} \times 10^{6} \\
& \approx\left\{\left(\sigma_{\mathrm{R}}-\sigma_{\mathrm{S}}\right)-\frac{4}{3} \pi\left(\chi_{\mathrm{R}}-\chi_{\mathrm{S}}\right)\right\} \times 10^{6}
\end{aligned}
$$

Here, $\omega$ and $\sigma$ are the Larmor frequency and the shielding constant, respectively. The genuine chemical shift, $\delta_{\text {genuine }}$, is defined by the difference of the shielding constants, which can be correlated with the observed one as follows by using Eq. (3).

$$
\begin{aligned}
\delta_{\text {genuine }} & \equiv\left(\sigma_{\mathrm{R}}-\sigma_{\mathrm{S}}\right) \times 10^{6} \\
& =\delta_{\text {obs }}+\frac{4}{3} \pi\left(\chi_{\mathrm{R}}-\chi_{\mathrm{S}}\right) \times 10^{6}
\end{aligned}
$$


The volume magnetic susceptibilities of heavy water and PTFE are $\chi_{\mathrm{R}}=-0.719 \times 10^{-6}$ and $\chi_{\mathrm{s}}=-0.836 \times 10^{-6}$ in the cgs unit, respectively. ${ }^{13}$ Therefore, the second term of Eq. (4) is calculated to be $+0.57 \mathrm{ppm}$, and the genuine chemical shift is thus corrected to be ca. $2.1 \mathrm{ppm}$.

The chemical shift of proton responds to the chemical environment. In the present case, the dipole-dipole interaction is the major chemical factor to make the water molecules adsorbed on the PTFE surface, since no ionic species is available. The peak position responds to the strength of the electron-attractive interaction. ${ }^{14-17}$ In a previous work, the chemical shift of proton of water dissolved in various polar and non-polar organic solvents is reported. ${ }^{16,17}$ The representatives are $2.8,2.1,1.6$, and $1.1 \mathrm{ppm}$ in acetone, acetonitrile, chloroform, and carbon tetrachloride, respectively, at $30^{\circ} \mathrm{C}$. The results show an apparent tendency that the chemical shift moves to a lower magnetic field when the polar character of the solvent becomes stronger. The peak position of $2.1 \mathrm{ppm}$ in the present study is same as that of water dissolved in acetonitrile that has a large dipole moment. ${ }^{18,19}$ This strongly indicates that the adsorbed water molecules are stuck to a disaggregated PTFE molecule having a strong dipole character, which further suggests that the adsorption should be fairly strong.

Analysis of molecular motion of the adsorbed water: If the adsorbed water molecules are strongly attached to the disaggregated PTFE molecule, the motion of water should strongly be restricted. Thus, the spin-lattice relaxation time, $T_{1}$, of the adsorbed water molecules was measured by using the inversion recovery method ${ }^{11}$ As a total, 18 spectra were recorded with different mixing time $\left(t_{\text {mix }}\right)$ varied from $10 \mu$ s to $10 \mathrm{~s}$.

Figure 3 presents the ${ }^{1} \mathrm{H}$ NMR spectra of the stretched PTFE tape in a vacuum by the inversion recovery method. The sample tape was pretreated with a saturated water vapor for 72 $\mathrm{h}$, which is the same as that for the orange spectrum in Figure 2. Since the baseline curvature has 
nothing to do with the adsorbed-water peak, the baseline was removed by subtracting the NMR spectrum of dry air. As a result, the intensity of the peak at $1.5 \mathrm{ppm}$ in Figure 2 is plotted against $t_{\text {mix }}$ as presented in Figure 4 with confirming the reproducibility. The plot was readily fitted by using a single exponential function with a time constant of $T_{1}=0.557 \pm 0.001 \mathrm{sec}$.

With the $T_{1}$ value, the rotational correlation time, $\tau_{c}$, is obtained by using the Bloembergen-Purcell-Pound (BPP) theory ${ }^{20,21}$ as presented by Eq. (5). The BPP equation theorizes the random motion of the dipole-dipole interaction between the two protons of water to induce the magnetic relaxations,

$$
R_{1}=\frac{1}{T_{1}}=\left(\frac{\mu_{0}}{4 \pi}\right)^{2}\left(\frac{3 \hbar^{2} \gamma^{4}}{10 r^{6}}\right)\left(\frac{\tau_{\mathrm{c}}}{1+\omega_{0}^{2} \tau_{\mathrm{c}}^{2}}+\frac{4 \tau_{\mathrm{c}}}{1+4 \omega_{0}^{2} \tau_{\mathrm{c}}^{2}}\right)
$$

Here, $\mu_{0}$ is the magnetic permeability in vacuum, $\gamma$ is the gyromagnetic ratio of proton, $r$ is the distance between two protons in a water molecule, and $\omega_{0}$ is the Larmor frequency. By solving the equation for $T_{1}$, the correlation time, $\tau_{\mathrm{c}}$, is obtained. The inter-nuclear distance, $r$, was fixed to $0.151 \mathrm{~nm}$ by considering the typical value of gaseous water. ${ }^{22,23}$ When putting the $T_{1}$ value of $0.557 \mathrm{sec}$ in the equation, $\tau_{\mathrm{c}}$ is obtained to be $44 \mathrm{~ns}$, which is four orders of magnitude larger than that of pure liquid water $\left(2.6\right.$ ps at $\left.25^{\circ} \mathrm{C}\right) .{ }^{23}$ This means that the adsorbed water molecules on a disaggregated PTFE molecule are highly restricted in motion.

In a previous study, $\tau_{\mathrm{c}}$ of hydration water about an ionized sulfonate group in a Nafion ${ }^{\circledR}$ membrane was analyzed in a similar manner. The first and second hydration layers about the sulfonate group have water molecules exhibiting $\tau_{\mathrm{c}}$ of 8.8 and $220 \mathrm{~ns}$, respectively. ${ }^{22}$ Therefore, $^{2}$ $\tau_{\mathrm{c}}=44 \mathrm{~ns}$ is concluded to be a fairly long correlation time, which implies that the motion of the adsorbed water molecule is highly restricted, as if it were near an ionized group. In this manner, 
the spontaneous adsorption of water from ambient air onto the disaggregated Rf group, which is induced by stretching a PTFE tape, occurs very strongly as expected by the SDA theory.

Thermal desorption of water: If the strong adsorption is truly correct, the adsorbed water molecules should be highly stable on the PTFE tape, and they would also be stable against a sample heating. To confirm the thermal stability, NMR spectra of the water-adsorbed PTFE tape were measured at elevated temperatures up to $250{ }^{\circ} \mathrm{C}$ in a vacuumed condition (the same condition as for the red spectrum in Figure 2).

The spectra are presented in Figure 5. Almost no change is found even when the sample is heated up to $100{ }^{\circ} \mathrm{C}$. If the PTFE surface is hydrophobic, the water should leave the surface spontaneously even near the room temperature. Therefore, the thermal stability is concluded to be unusually high, which agrees with the analytical results in the previous sections. When the sample is heated up to $150{ }^{\circ} \mathrm{C}$, the decrease of adsorbed water is apparently recognized. When the sample is heated at $200^{\circ} \mathrm{C}$, the amount of the remained water on the PTFE surface becomes largely decreased.

\section{CONCLUSION}

A stretched PTFE tape is found to attract molecular water on the surface via the dipole-dipole interaction as a result of a collapse of the SDA structure in the polymer aggregate after the mechanical stress on the tape. The adsorbed water yields an extraordinarily large rotational correlation time to that of free water, which straightforwardly indicates that the adsorbed water molecules are highly restricted in motion. The thermal desorption experiments support the strong interaction between the molecular water and the surface of a stretched PTFE tape. Since an unstretched PTFE tape exhibits no water adsorption, the interaction between a single Rf group and molecular water after the collapse of the SDA packing of polymeric molecules by the mechanical 
stress is the key to understand the attraction of molecular water. Since a water droplet exhibited ignorable difference in the contact angles on the un-stretched and stretched tapes, the disaggregated Rf group proved to strongly attract "molecular water" selectively. In this manner, the discrimination between the molecular and bulky water has been found very important to discuss the hydrophobicity of a single Rf group and the surface of a bulky Rf compound.

\section{AUTHOR INFORMATION}

\section{Corresponding Author}

*E-mail: htakeshi@scl.kyoto-u.ac.jp

\section{ACKNOWLEDGMENT}

This work was financially supported by Grant-in-Aid for Scientific Research (A) (No. $15 \mathrm{H} 02185$ (TH)) from Japan Society for the Promotion of Science, for which the authors thanks are due.

\section{REFERENCES}

1. Krafft, M. P.; Riess, J. G. Chemistry, Physical Chemistry, and Uses of Molecular Fluorocarbon-Hydrocarbon Diblocks, Triblocks, and Related Compounds-Unique “Apolar” Components for Self-Assembled Colloid and Interface Engineering. Chem. Rev. 2009, 109, 1714-1792.

2. Hasegawa, T.; Shimoaka, T.; Shioya, N.; Morita, K.; Sonoyama, M.; Takagi, T.; Kanamori, T. Stratified Dipole-Arrays Model Accounting for Bulk Properties Specific to Perfluoroalkyl Compounds. ChemPlusChem 2014, 79, 1421- 1425. 
3. Hasegawa, T. Understanding of the Intrinsic Difference between Normal- and PerfluoroAlkyl Compounds toward Total Understanding of Material Properties. Chem. Phys. Lett. 2015, 627, 64-66.

4. London, F. The General Theory of Molecular Forces. Trans. Faraday Soc. 1937, 8-26.

5. Kim, H. K.; Shi, F. G. Refractive Index of Polycrystalline Submicrometer Polymer Thin Films: Thickness Dependence. J. Mat. Sci. Mat. Electronics 2001, 12, 361-364.

6. Chessick, J. J.; Healey, F. H.; Zeitlemoyer, A. C. Adsorption and Heat of Wetting Studies of Teflon. J. Phys. Chem. 1956, 60, 1345-1347.

7. Fowkes, F. M.; McCarthy, D. C.; Mostafa, M. A. Contact Angle and the Equilibrium Spreading Pressures of Liquids on Hydrophobic Solids. J. Colloid Interface Sci. 1980, 78, 200-206.

8. Dalvia, V. H.; Rossky, P. J. Molecular Origins of Fluorocarbon Hydrophobicity. Proc. Nat. Acad. Sci. 2010, 107, 13603-13607.

9. Lee, M. W.; Latthe, S. S.; Tarin, A. L.; Yoon, S. S. Dynamic Electrowetting-on-Dielectric on Unstretched and Stretched Teflon. Langmuir, 2013, 29, 7758-7767.

10. Weisensee, P. B.; Neelakantan, N. K.; Suslick, K. S.; Jacobi, A. M.; King, W. P. Impact of Air and Water Vapor Environments on the Hydrophobicity of Surfaces. J. Colloid Interface Sci. 2015, 453, 177-185.

11. Carr, H. Y.; Purcell, E. M. Effects of Diffusion on Free Precession in Nuclear Magnetic Resonance Experiments. Phys. Rev. 1954, 94, 630-638. 
12. Becker, E. D. High Resolution NMR Theory and Chemical Applications, Academic Press, New York, 1980.

13. Doty, F. D.; Entzminger, G.; Yang, Y, A. Magnetism in High-Resolution NMR Probe Design I: General Methods. Concepts in Magn. Reson. 1998, 10, 133-156.

14. Schneider, W. G.; Bernstein, H. J.; Pople, J. A. Proton Magnetic Resonance Chemical Shift of Free (Gaseous) and Associated (Liquid) Hydride Molecules. J. Chem. Phys. 1958, 28, 601-607.

15. Akitt, J. W. Proton Chemical Shifts for Water in Cationic Hydration Complexes and their Contribution to Water Shifts in Electrolyte Solution. J. Chem. Soc. Dalton Trans. 1973. 1, 42-49.

16. Wakai, C. NMR Studies on Rotational Dynamics of Water and Benzene Molecules in Organic Solvents. Master Thesis, Kyoto Univ. 1993.

17. Nakakaha, M.; Wakai, C. Monomeric and Cluster States of Water Molecules in Organic Solvent. Chem. Lett. 1992, 809-812.

18. Steiner, P. A.; Gordy, W. Precision Measurement of Dipole Moments and Other Spectral Constants of Normal and Deuterated Methyl Fluoride and Methyl Cyanide. J. Mol. Spectrosc. 1966, 21, 291-301.

19. Desfrançois, C.; Abdoul-Carime, H.; Adjouri, C.; Khelifa, N.; Schermann, J. P. Dipole Binding to a Strongly Polar Molecule and its Homogeneous Clusters: Magnetic Distribution of Acetonitrile Cluster Anions. Eur. Lett. 1994, 26, 25-30. 
20. Bloembergen, N.; Purcell, E. M.; Pound, R. V. Relaxation Effects on Nuclear Magnetic Resonance Absorption. Phys. Rev. 1948, 73, 679-715.

21. Abragam, A. The principle of nuclear magnetism, Oxford University Press, New York, 1961.

22. Wakai, C.; Shimoaka, T.; Hasegawa, T. Analysis of the Hydration Process and Rotational Dynamics of Water in a Nafion Membrane Studied by ${ }^{1} \mathrm{H}$ NMR Spectroscopy. Anal. Chem. 2013, $85,7581-7587$.

23. Lankhorst, D.; Schriever, J.; Leyte, J. C. Determination of the Rotational Correlation Time of Water by Proton NMR Relaxation in $\mathrm{H}_{2}{ }^{17} \mathrm{O}$ and Some Related Results. Ber. Bunsenges. Phys. Chem. 1982, 86, 215-221. 


\section{FIGURE CAPTIONS}

Figure 1 A schematic of rolled PTFE tapes in a NMR tube. To make the shape factor be zero, three tapes are piled up to have a cylindrical shape. If needed, a small water vessel is hung in the tube.

Figure $2 \quad{ }^{1}$ H NMR spectra of PTFE tapes with various conditions: (a) un-stretched tape stored in ambient air (dark-blue), (b) stretched tape stored in ambient air (green), (c) stretched tape with saturated water gas for 3 days (orange), (d) the tape (c) is put in a vacuumed condition for $14 \mathrm{~h}$ (red), and (e) dry air as reference (black).

Figure $3 \quad{ }^{1} \mathrm{H}$ NMR spectra of a stretched PTFE tape in a vacuumed condition for $14 \mathrm{~h}$ measured by the inversion recovery method dependent on $t_{\text {mix }}$ of $10 \mu \mathrm{s}, 1 \mathrm{~ms}, 2 \mathrm{~ms}$, $3 \mathrm{~ms}, 5 \mathrm{~ms}, 7 \mathrm{~ms}, 9 \mathrm{~ms}, 11 \mathrm{~ms}, 13 \mathrm{~ms}, 15 \mathrm{~ms}, 20 \mathrm{~ms}, 40 \mathrm{~ms}, 100 \mathrm{~ms}, 250 \mathrm{~ms}, 750 \mathrm{~ms}$, $2 \mathrm{~s}, 4 \mathrm{~s}$ and $10 \mathrm{~s}$ in the upward direction.

Figure 4 Analysis of $T_{1}$ by fitting an exponential curve to the peak intensities read from the NMR spectra in Figure 3 after subtraction of the baselines.

Figure 5 Temperature dependent ${ }^{1} \mathrm{H}$ NMR spectra of the stretched PTFE tape in vacuum. The temperatures are at $30,50,100,150,200$ and $250{ }^{\circ} \mathrm{C}$, in the downward direction. A reference spectrum of dry air is also overlaid. 


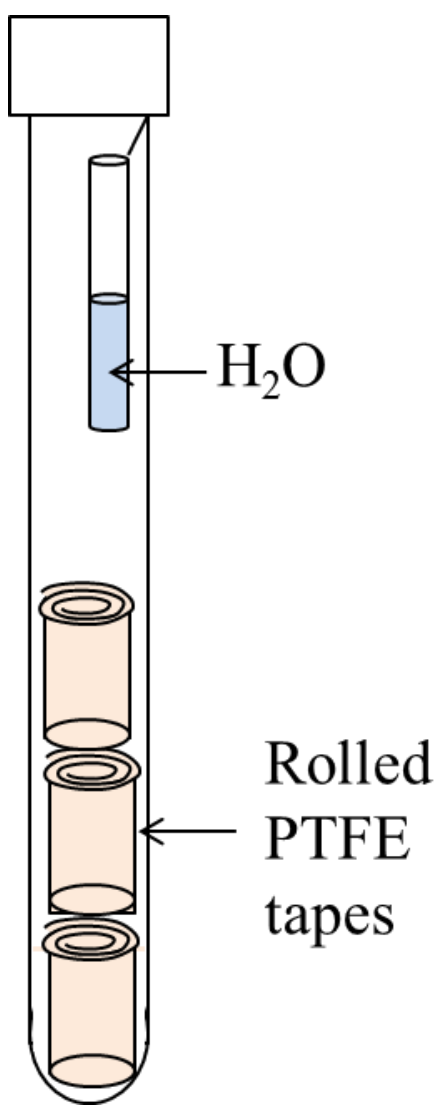

Figure 1 C. Wakai et al. 


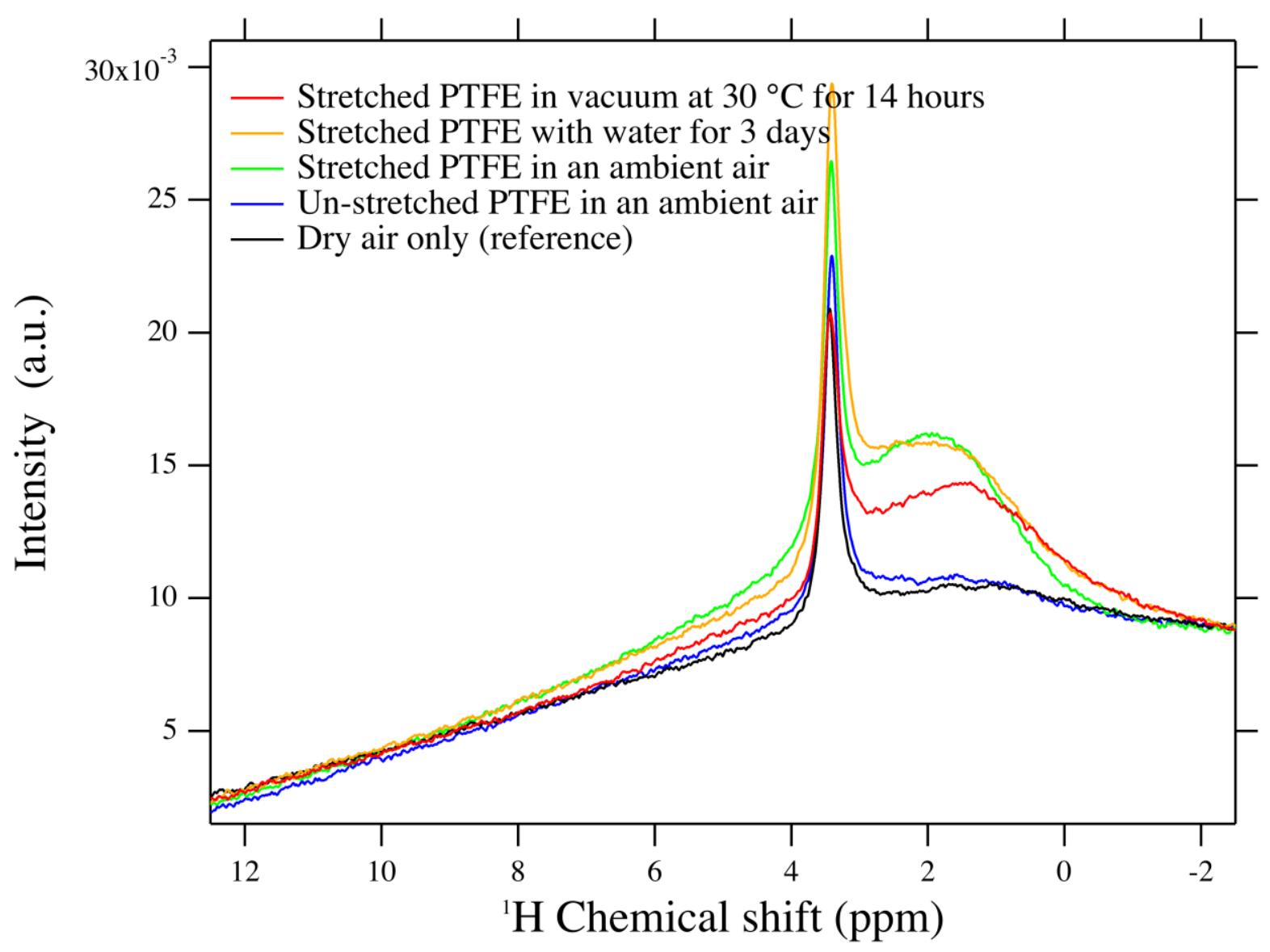

Figure 2 C. Wakai et al. 


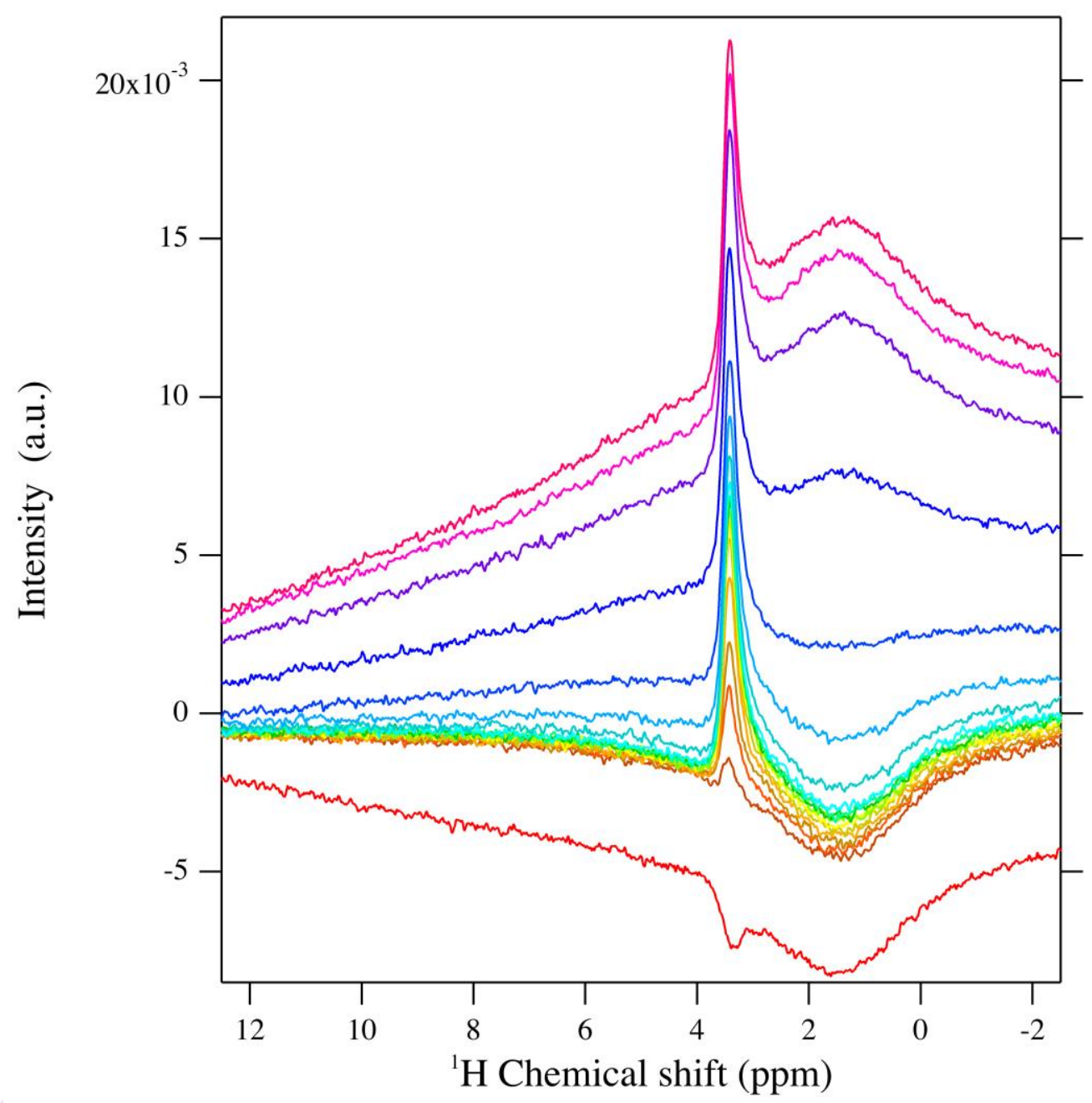

Figure 3 C. Wakai et al. 


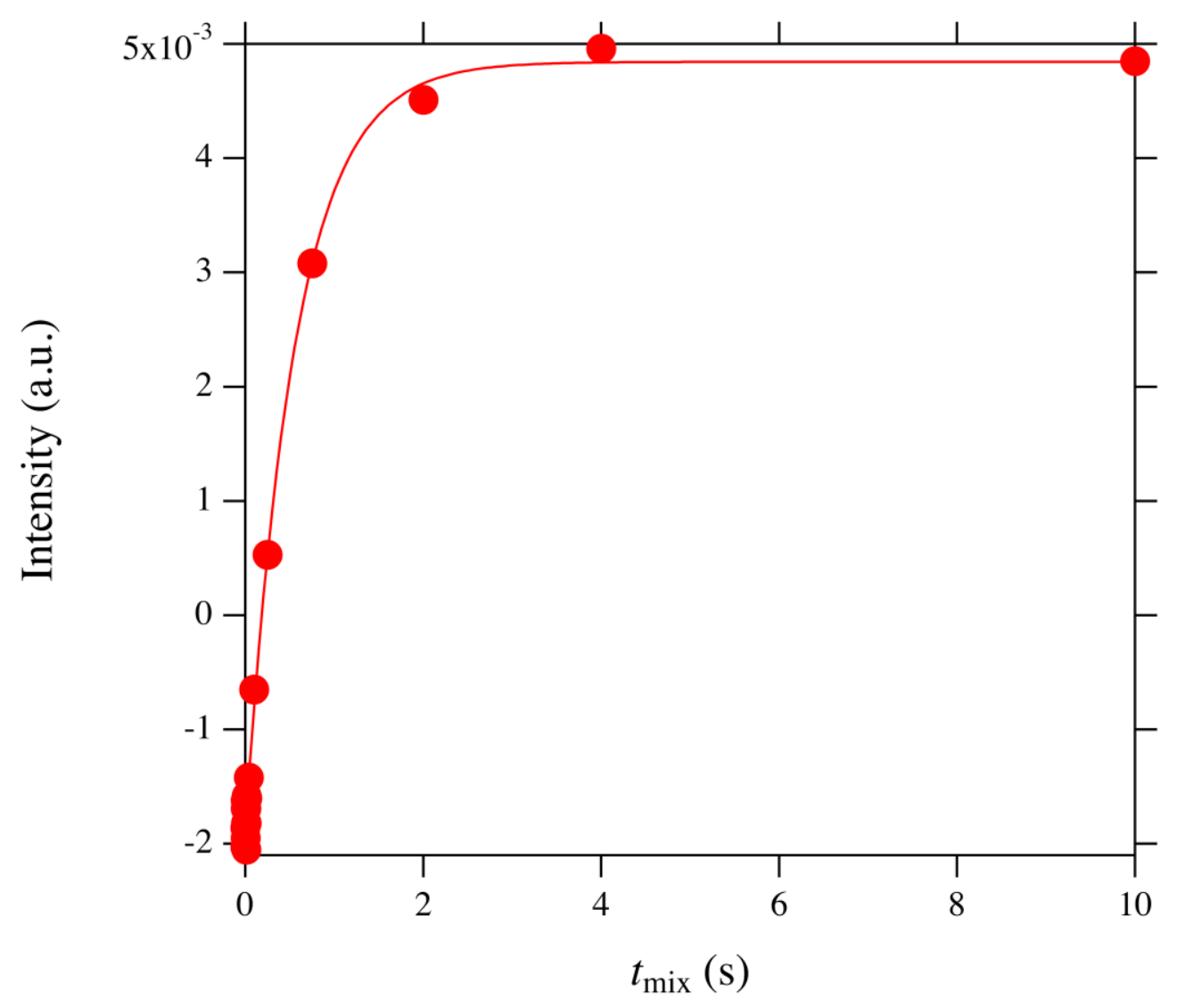

Figure 4 C. Wakai et al. 


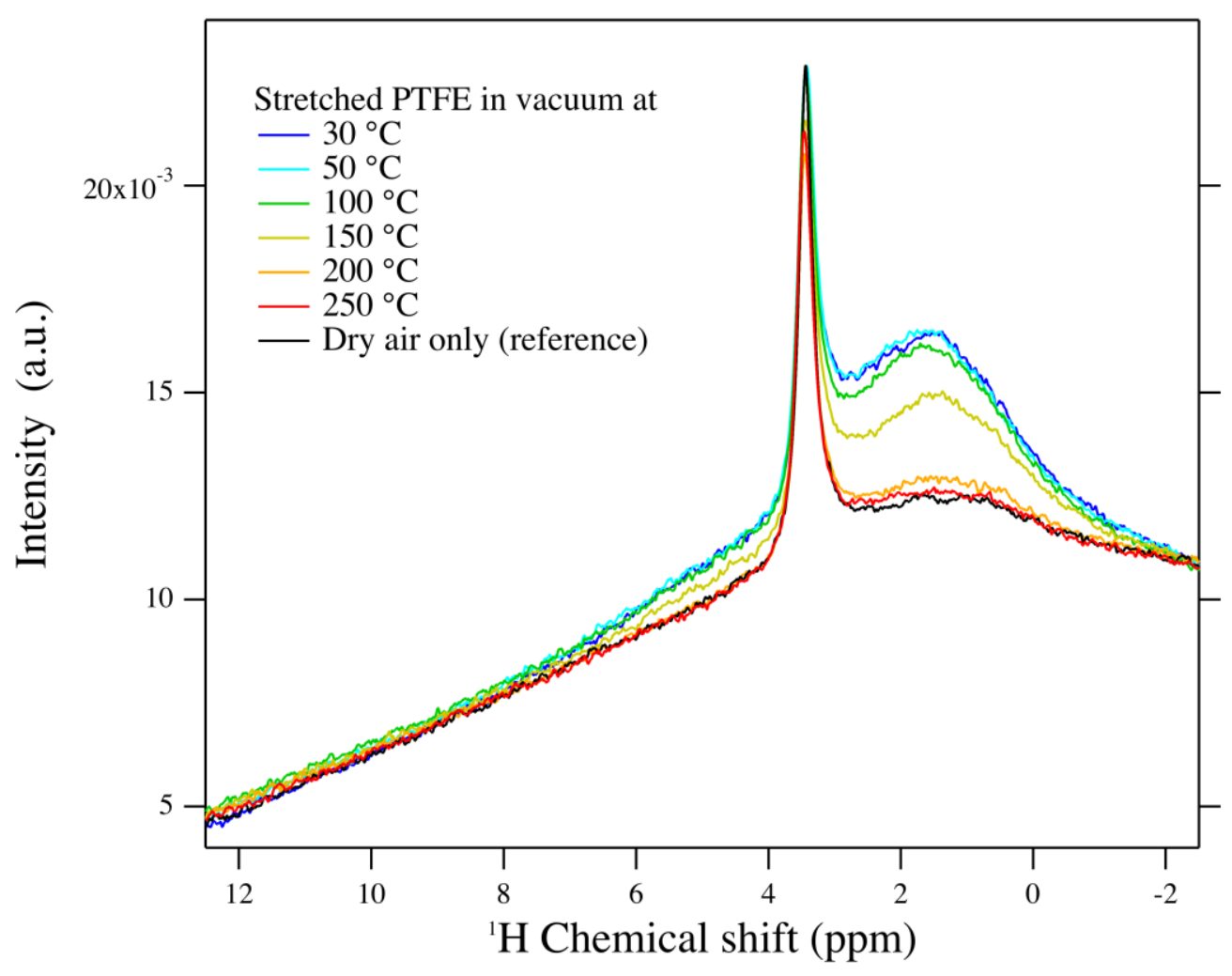

Figure 5 C. Wakai et al. 


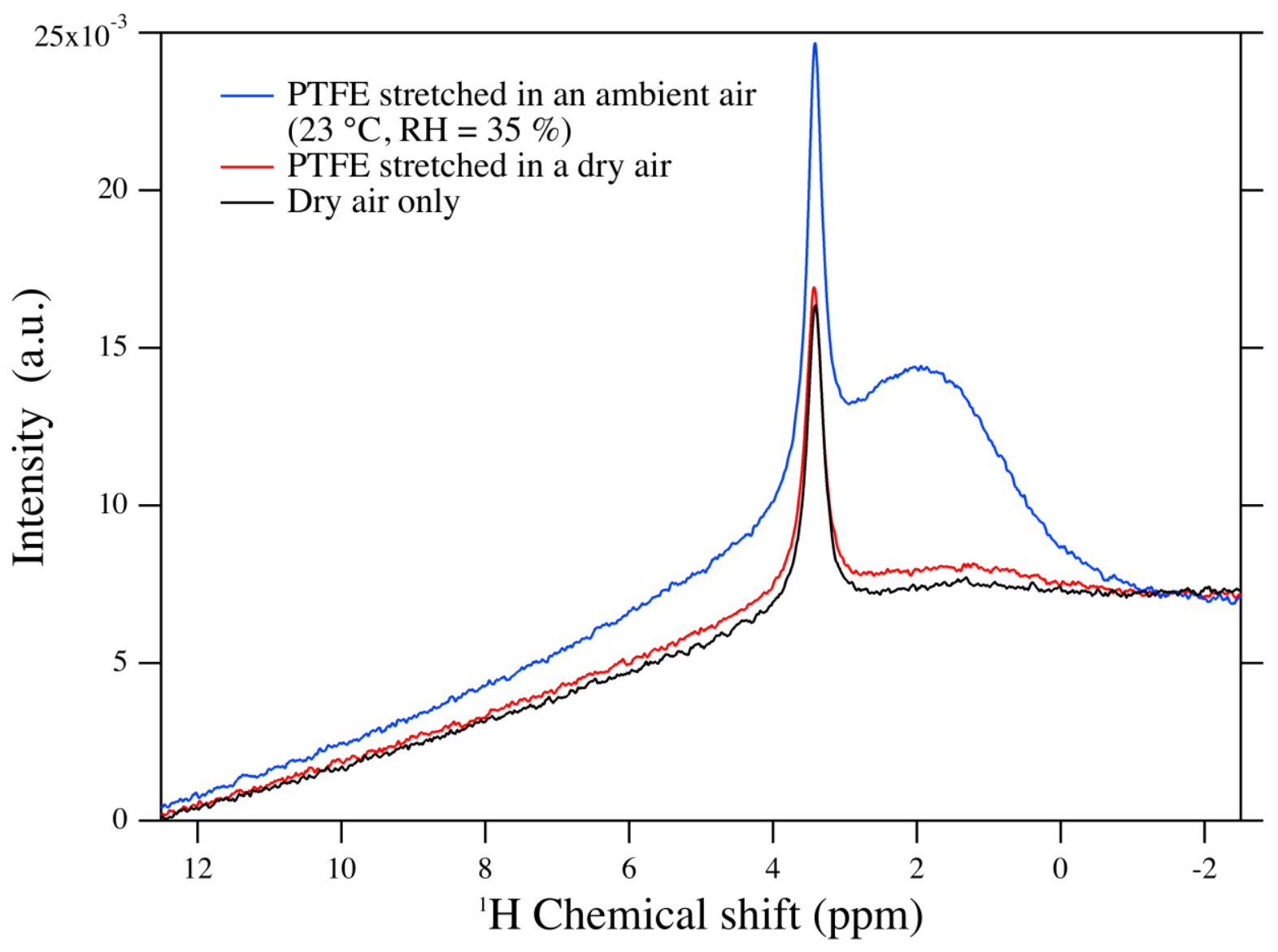

Figure S1 ${ }^{1} \mathrm{H}$ spectra for PTFE tapes stretched in (a) an ambient air and (b) a dry air. The spectrum for a dry air only is a reference. 


\section{TOC graphics}
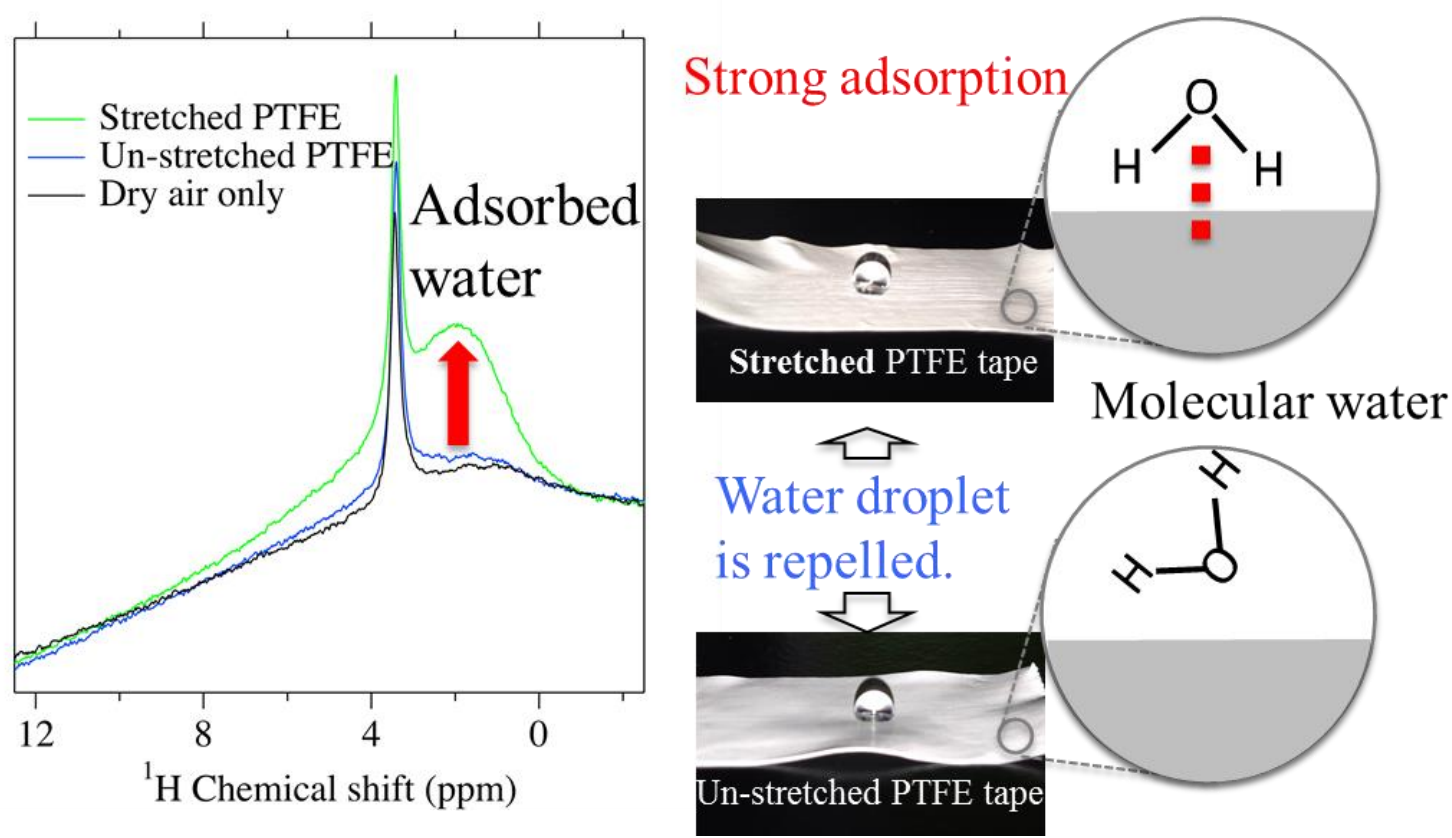